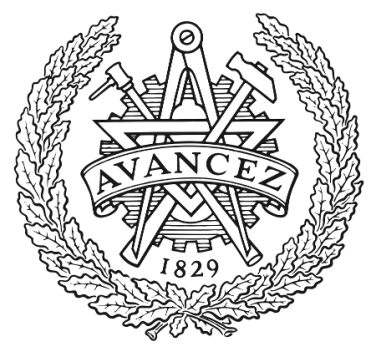

CHALMERS

UNIVERSITY OF TECHNOLOGY

\title{
Thermovoltage in quantum dots with attractive interaction
}

Downloaded from: https://research.chalmers.se, 2023-04-26 12:47 UTC

Citation for the original published paper (version of record):

Schulenborg, J., Wegewijs, M., Splettstoesser, J. (2020). Thermovoltage in quantum dots with attractive interaction. Applied Physics Letters, 116(24). http://dx.doi.org/10.1063/5.0008866

N.B. When citing this work, cite the original published paper. 


\section{Thermovoltage in quantum dots with attractive interaction}

Cite as: Appl. Phys. Lett. 116, 243103 (2020); https://doi.org/10.1063/5.0008866

Submitted: 26 March 2020 . Accepted: 31 May 2020 . Published Online: 16 June 2020

(D) Jens Schulenborg, (D) Maarten R. Wegewijs, and (iD) Janine Splettstoesser

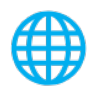

\section{ARTICLES YOU MAY BE INTERESTED IN}

An elementary photo-thermoelectric transistor: Experimental demonstration Applied Physics Letters 116, 243501 (2020); https://doi.org/10.1063/5.0010264

Niobium Dayem nano-bridge Josephson gate-controlled transistors Applied Physics Letters 116, 242601 (2020); https://doi.org/10.1063/5.0011304

Spin-orbit torque field-effect transistor (SOTFET): Proposal for a magnetoelectric memory Applied Physics Letters 116, 242405 (2020); https://doi.org/10.1063/5.0002909

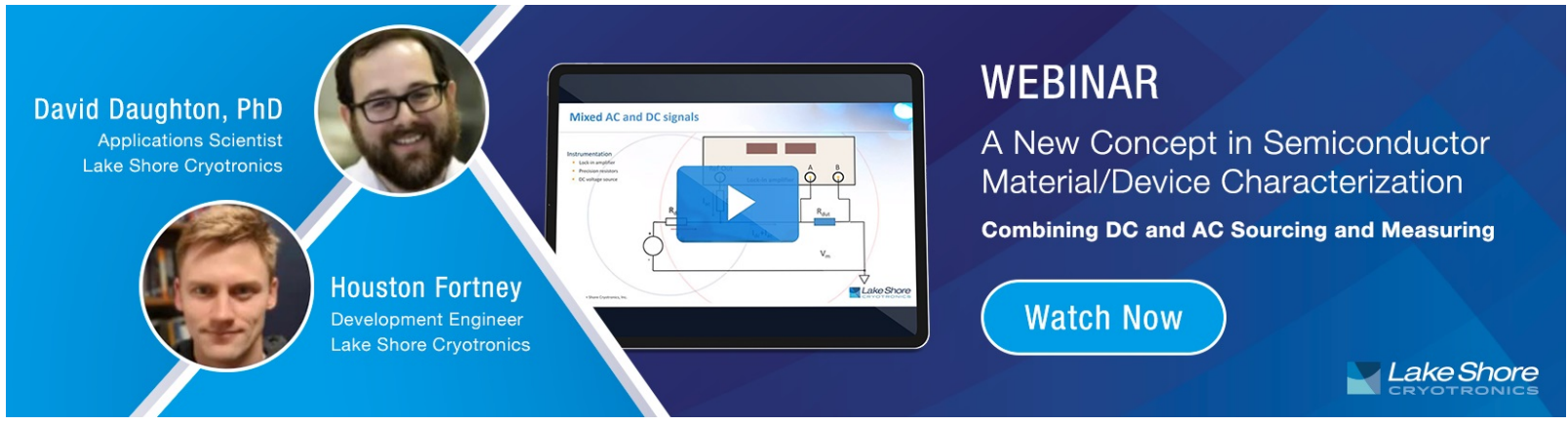




\title{
Thermovoltage in quantum dots with attractive interaction
}

\author{
Cite as: Appl. Phys. Lett. 116, 243103 (2020); doi: 10.1063/5.0008866 \\ Submitted: 26 March 2020 - Accepted: 31 May 2020 . \\ Published Online: 18 June 2020

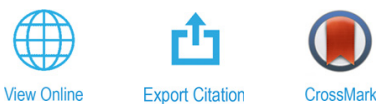

Jens Schulenborg, $^{1,2, a)}$ (D) Maarten R. Wegewijs, ${ }^{3,4}$ (D) and Janine Splettstoesser ${ }^{2}$ (iD

\begin{abstract}
AFFILIATIONS
${ }^{7}$ Center for Quantum Devices, Niels Bohr Institute, University of Copenhagen, 2100 Copenhagen, Denmark

${ }^{2}$ Department of Microtechnology and Nanoscience, Chalmers University of Technology, 41296 Göteborg, Sweden

${ }^{3}$ Institute for Theory of Statistical Physics, RWTH Aachen, 52056 Aachen, Germany

${ }^{4}$ Peter Grünberg Institut and JARA, Forschungszentrum Jülich, 52425 Jülich, Germany
\end{abstract}

${ }^{\text {a) }}$ Author to whom correspondence should be addressed: jens.schulenborg@nbi.ku.dk

\begin{abstract}
We study the linear and nonlinear thermovoltage of a quantum dot with effective attractive electron-electron interaction and weak, energydependent tunnel coupling to electronic contacts. Remarkably, we find that the thermovoltage shows signatures of repulsive interaction, which can be rationalized. These thermovoltage characteristics are robust against large potential and temperature differences well into the nonlinear regime, which we expect can be demonstrated in current state-of-the-art experiments. Furthermore, under nonlinear operation, we find extended regions of large power production at efficiencies on the order of the Curzon-Ahlborn bound interrupted only by a characteristic sharp dip.
\end{abstract}

Published under license by AIP Publishing. https://doi.org/10.1063/5.0008866

Recently, different types of devices with an effectively attractive electron-electron interaction ${ }^{1}$ have been experimentally investigated ${ }^{2}$ and quantum dot structures with attractive onsite-interaction have also been realized. ${ }^{3,4}$ In these quantum dots, signatures of pair tunneling $^{5-7}$ induced by the attractive onsite interaction could be identified in transport properties.

In the present letter, we predict surprising features in the thermovoltage of such quantum dots. We show that the linear-response thermovoltage-the Seebeck coefficient-shows signatures at quantum-dot level positions that are characteristic of Coulomb oscillations due to repulsive onsite interaction. We rationalize this fact and show that it can be exploited in an analysis of how these features are modified under various realistic experimental conditions. The discussed effects are highly relevant for the characterization of attractive systems, which has only recently started.

Simultaneously, there has been significant progress in investigating linear and nonlinear response thermoelectrics in quantum dot devices (see Refs. 8-20 and references therein). These are of interest for on-chip energy harvesting, and their Seebeck coefficient is a key parameter to characterize them. Our analysis instead reveals the thermoelectric properties of systems with strong attractive electron-electron interaction. We also explicitly address energy-dependent tunnel couplings between the dot and the environment as energy filters in addition to the quantum-dot levels. Efficient nanoscale thermoelectrics, in particular three-terminal energy harvesters, ${ }^{10,21-24}$ crucially rely on this energy-dependent coupling. In this Letter, we characterize the performance of such quantum dots with attractive interaction as steady-state heat engines and find extended regions of large power production and efficiency.

Finally, the thermoelectric response of repulsive quantum $\operatorname{dots}^{16,19}$ has successfully been analyzed using a mapping based on a fermionic duality relation, ${ }^{25}$ providing simple analytical formulas. Here, this relation enables us to explain the thermoelectric response of a quantum dot with attractive interaction in terms of the well understood physics of a repulsive dot. This simple description can serve as a guide for future experiments.

The quantum dot of interest is sketched in Fig. 1(a). It is modeled as a single spin-degenerate level, with an attractive electron-electron interaction. We assume the level spacing to be large compared to any other energy scale relevant for transport, such as voltage bias and temperatures; indeed, recent experimental realizations of quantum dots with attractive interactions have been well explained in terms of such a model. ${ }^{4}$ The isolated dot is then described by the Hamiltonian,

$$
H=\tilde{\varepsilon} N-|U| N_{\uparrow} N_{\downarrow},
$$

with $\tilde{\varepsilon}$ being the single-level energy, $N=N_{\uparrow}+N_{\downarrow}$ the local particlenumber operator with spin-resolved components $N_{\uparrow}$ and $N_{\downarrow}$, and 

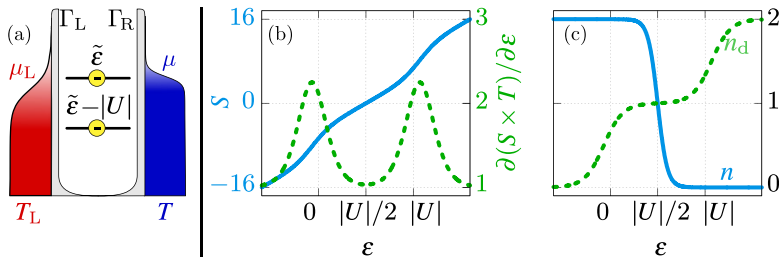

FIG. 1. (a) Sketch of a quantum dot with attractive interaction. (b) Seebeck coefficient $S$ (blue solid) and its derivative (green dashed) and (c) equilibrium charge $n$ and its dual $n_{\mathrm{d}}$, as a function of dot level $\varepsilon=\tilde{\varepsilon}-\mu$. We take $T=|U| / 10$ and $\Gamma_{\mathrm{L}, \mathrm{R}}$ that are energy-independent.

$|U|=-U$ the interaction strength. The quantum dot is tunnelcoupled to two electronic reservoirs, $\alpha=\mathrm{L}, \mathrm{R}$, with electrochemical potentials $\mu_{\mathrm{L}}=\mu-V$ and $\mu_{\mathrm{R}}=\mu$ and temperatures $T_{\mathrm{L}}=T+\Delta T$ and $T_{\mathrm{R}}=T$. We assume the experimentally relevant case of weak tunnel rates $\Gamma_{\alpha}(E) \ll T_{\alpha}$, implying that pair tunneling is enabled by thermal excitations. ${ }^{4,26,27}$ We allow these rates to strongly depend on the energy of the tunneling process. We set $k_{\mathrm{B}}=\hbar=|e|=1$.

We analyze the full thermoelectric response of the quantum dot using expressions obtained from a recently established, general fermionic duality. ${ }^{16,19,25,28}$ This purely dissipative symmetry, applied to a weak-coupling master-equation description, maps the transport dynamics of fermionic open nonequilibrium systems to those of $d u a l$ systems with sign-inverted local energies, chemical potentials, and energy dependencies of the tunnel couplings, replacing $\left[\tilde{\varepsilon},-|U|, \mu_{\alpha}\right.$, $\left.\Gamma_{\alpha}(E)\right] \rightarrow\left[-\tilde{\varepsilon},|U|,-\mu_{\alpha}, \Gamma_{\alpha}(-E)\right.$.] In the present case, the remarkable duality enables us to predict nonequilibrium effects in the thermoelectric response of the attractive dot of interest by relating them to the properties of the quantum dot with equilibrium parameters and attractive interaction as well as to its dual model with repulsive interaction. ${ }^{29}$ The key quantities in this analysis are the equilibrium dot occupation for attractive interaction,

$$
n(\varepsilon,-|U|)=\langle N\rangle=\frac{2 f(\varepsilon)}{1+f(\varepsilon)-f(\varepsilon-|U|)},
$$

with $\varepsilon=\tilde{\varepsilon}-\mu$ and $f(x)=[\exp (x / \mathrm{T})+1]^{-1}$ the Fermi function, and most importantly, the dual occupation, ${ }^{19}$

$$
n_{\mathrm{d}}=n(-\varepsilon,|U|)=\frac{2[1-f(\varepsilon)]}{1-f(\varepsilon)+f(\varepsilon-|U|)},
$$

to which the duality assigns a repulsive interaction $|U|$. Figure 1 (c) shows both $n$ and $n_{\mathrm{d}}$ as functions of $\varepsilon$.

We start with the linear response of the thermovoltage, for small $V$ and $\Delta T$, and the linear Seebeck coefficient $S=V /\left.\Delta T\right|_{I=0}$ at vanishing charge current $I=0$ [see Eq. (10)] across the dot and first consider energy-independent tunnel couplings $\Gamma_{\alpha}(E) \rightarrow \Gamma_{\alpha}$. The explicit formula for $S$ has a remarkably simple form ${ }^{16}$ in terms of the dual dot occupation (3),

$$
S \times T=\varepsilon-|U|\left(2-n_{\mathrm{d}}\right) / 2 .
$$

The consequences of the attractive interaction are shown in Fig. 1(b). On the one hand, we find a linear $\varepsilon$-dependence $S \times T \approx \varepsilon-|U| / 2$ around the zero-crossing at $\varepsilon=|U| / 2$. This is intuitively expected in analogy to the well-known case of repulsive quantum dots: $:^{16,30-32}$ as reflected by $n(\varepsilon)$ in Fig. 1(c), the attractive dot effectively acts as a single resonance at $\varepsilon=|U| / 2$. On the other hand, we find $S \times T$ $\rightarrow \varepsilon-|U|$ for $\varepsilon<0$ and $S \times T \rightarrow \varepsilon$ for $\varepsilon>|U|$. Here, the attractive interaction does not anymore favor thermally excited pair transitions over single-electron transitions. The resulting crossovers between all three identified regimes lead to surprising kinks in the Seebeck coefficient $S(\varepsilon)$ [blue line in Fig. 1(b)]. These kinks are even better visible in its derivative ${ }^{33}$ [green, dashed line in Fig. 1(b)], measurable using lockin techniques,

$$
T \times \frac{\partial S}{\partial \varepsilon}=1+\delta n_{\mathrm{d}}^{2} \times \frac{|U|}{2 T} .
$$

Indeed, this derivative depends on the equilibrium charge fluctuations $\delta n_{\mathrm{d}}^{2}=\left\langle N^{2}\right\rangle_{\mathrm{d}}-n_{\mathrm{d}}^{2}$ after the duality mapping. This implies features in $S(\varepsilon)$ at the Coulomb resonances $\varepsilon=0$ and $\varepsilon=-U=|U|$ of a repulsive dot. The appearance of the dual occupation $n_{\mathrm{d}}$ in Eq. (4) is not expected from a brute-force "Fermi's Golden rule" calculation and defies common physical intuition. This is typical for insights offered by fermionic duality. ${ }^{16,19,25}$

The mapping to a repulsive system via $n_{\mathrm{d}}$ enables a further prediction for experiments. The peaks in $\partial S / \partial \varepsilon$ are shifted by approximately $\pm T \ln (2)$ away from the zero-temperature resonances $\varepsilon=0,|U|$ of a repulsive dot. In the latter case, this shift is well understood as the entropy of the singly occupied state due to the spin degeneracy. ${ }^{34-36}$ In an attractive dot, single occupation is never a stable equilibrium state, but remarkably, our dual picture reveals that its spin degeneracy, nevertheless, affects the Seebeck coefficient.

A relevant question is how energy-dependent couplings $\Gamma_{\alpha}(E)$ affect the thermovoltage. In experiments, the environment density of states may sizably vary around the Fermi energy and, thereby, give rise to such an energy dependency. Moreover, an appropriately tuned energy dependence can be beneficial for efficient nanoscale energy harvesting. . $17,21-23$

We account for this by assuming arbitrary smoothly energydependent rates $\Gamma_{\alpha=\mathrm{L}, \mathrm{R}}(E) \ll T$ within the weak-coupling constraint. Following Ref. 19, $S$ is then determined by

$$
S \times T=\varepsilon-\frac{(1+\Lambda)\left(2-n_{\mathrm{d}}\right)}{(1-\Lambda) n_{\mathrm{d}}+(1+\Lambda)\left(2-n_{\mathrm{d}}\right)}|U| .
$$

This introduces the energy asymmetry $\Lambda$ of the coupling,

$$
\Lambda=\frac{\Gamma_{U \mathrm{~L}} \Gamma_{U \mathrm{R}} \Gamma_{\varepsilon}-\Gamma_{\varepsilon \mathrm{L}} \Gamma_{\varepsilon \mathrm{R}} \Gamma_{U}}{\Gamma_{U \mathrm{~L}} \Gamma_{U \mathrm{R}} \Gamma_{\varepsilon}+\Gamma_{\varepsilon \mathrm{L}} \Gamma_{\varepsilon \mathrm{R}} \Gamma_{U}}
$$

with $\Gamma_{\varepsilon \mathrm{L}}=\Gamma_{\mathrm{L}}(\varepsilon), \Gamma_{\varepsilon \mathrm{R}}=\Gamma_{\mathrm{R}}(\varepsilon), \Gamma_{U \mathrm{~L}}=\Gamma_{\mathrm{L}}(\varepsilon-|U|), \Gamma_{U \mathrm{R}}=\Gamma_{\mathrm{R}}(\varepsilon-|U|)$, $\Gamma_{\varepsilon}=\Gamma_{\varepsilon \mathrm{L}}+\Gamma_{\varepsilon \mathrm{R}}$, and $\Gamma_{U}=\Gamma_{U \mathrm{~L}}+\Gamma_{U \mathrm{R}}$. The result for $S$ in the presence of energy-dependent tunnel coupling is shown in Fig. 2. Equation (6) enables us to systematically isolate how energy-dependent couplings $\Gamma_{\alpha}(E)$ influence the linear thermovoltage for different level positions $\varepsilon$ at fixed $U=-|U|$ and $T .^{37,47-50}$ In Fig. 2, we identify-as one main qualitative effect of energetic coupling asymmetry-a shift of the zerocrossing of $S$ as a function of $\varepsilon$ away from $\varepsilon_{0}=|U| / 2$. This shift, which we call $\Delta \varepsilon_{0}$, can be understood from Eq. (6). We exploit that well within $0<\varepsilon<|U|$ and for $|U| \gg T$, repulsive Coulomb blockade induces a plateau at $n_{\mathrm{d}} \approx 1$ in the dual occupation. This simplifies Eq. (6) to

$$
S \times T \rightarrow \varepsilon-(1+\Lambda)|U| / 2 \text { for } 0<\varepsilon<|U|,
$$



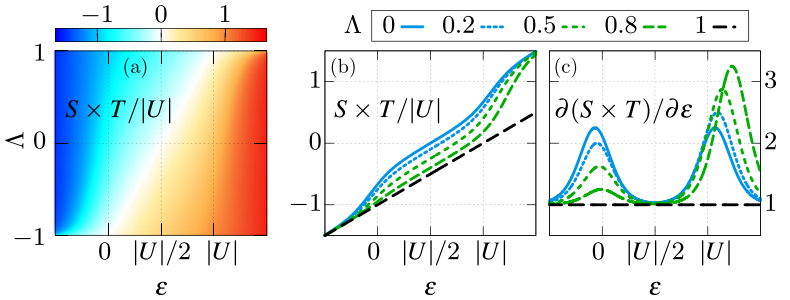

FIG. 2. Seebeck coefficient [(a) and (b)] and its derivative at fixed $\Lambda$ (c) as a function of dot level $\varepsilon$ and coupling-asymmetry $\Lambda$. We take energy-dependent $\Gamma_{\mathrm{L}}(E), \Gamma_{\mathrm{R}}(E)$, and $T=|U| / 10$.

implying that an asymmetry $\Lambda>0$ favors emission from a doubly occupied dot at addition energy $\varepsilon-|U|$ and $\Lambda<0$ favors absorption into an empty dot at energy $\varepsilon$. This offset of $S(\varepsilon)$ in Eq. (8) involves a zero crossing $\varepsilon_{0}$ shifted away from $|U| / 2$ by $\Delta \varepsilon_{0} \rightarrow|U| / 2 \times \Lambda$, predicting a pronounced effect for strong attractive interaction. The limit $\Delta \varepsilon_{0} \rightarrow \pm|U| / 2$ for $\Lambda \rightarrow \pm 1$ reflects that transport is reduced to a single resonance at $\varepsilon=0$ or $\varepsilon=|U|$, annulling all two-electron features.

Next, Fig. 2(c) demonstrates how the peaks in $\partial S / \partial \varepsilon$, and hence the kinks in $S$ visible in Fig. 1(b), change with energy-dependent couplings. For $\Lambda>0$, the peak in $\partial S / \partial \varepsilon$ around $\varepsilon=0$ shrinks with larger $|\Lambda|$, whereas the peak in $\partial S / \partial \varepsilon$ around $\varepsilon=|U|$ grows. At the same time, the latter also moves substantially further away from resonance-we refer to this shift from resonance as $\Delta \varepsilon_{\mathrm{p}}$. For $\Lambda<0$, the behavior is opposite with respect to the two peaks. In the singleresonance limit $|\Lambda| \rightarrow 1$, the slope $\partial S / \partial \varepsilon \times T \rightarrow 1$ becomes constant and both peaks disappear, as expected.

The change in the relative peak height follows from the offset of $S$ within $0<\varepsilon<|U|$ described in Eq. (8). For example, $\Lambda>0$, the shift to smaller $S$ in this $\varepsilon$-interval causes the step of $S(\varepsilon)$ around $\varepsilon=0$ to be smaller than around $\varepsilon=|U|$, see Fig. 2(b). This leads to a smaller relative peak height in $\partial S / \partial \varepsilon$.

The growing shift $\Delta \varepsilon_{\mathrm{P}}$ of the higher peak for increasing $|\Lambda|$ stems from the fact that the coupling asymmetry $\Lambda$ not only affects $S$ for $0<\varepsilon<|U|$ and large $|U| / T$ as in Eq. (8) but, in general, also influences where the crossover between the single-particle, linear limits $(S(\varepsilon) \sim \varepsilon$ and $S(\varepsilon) \sim \varepsilon-|U|)$ and the two-particle limit $(S(\varepsilon)$ $\sim \varepsilon-|U| / 2$ ) takes place [see Eq. (6)]. For both small $|\Lambda|$ and large $|\Lambda| \lesssim 1$, a useful analytical expression is [including the spindegeneracy shift $T \ln (2)]$

$$
\Delta \varepsilon_{\mathrm{P}} \approx T \times \operatorname{sgn}(\Lambda) \times \ln [2 /(1-|\Lambda|)] .
$$

For example, $\Lambda=0.8$ yields $\Delta \varepsilon_{\mathrm{p}} \approx 3.3 T \ln (2)$, which substantially deviates from the wideband limit result, where the shift away from resonance is given by $T \ln (2)$. In this case, $\Gamma_{U}$ is large enough compared to $\Gamma_{\varepsilon}$, such that even for a considerable interval with $\varepsilon>0$ and $\varepsilon-|U|>0$, the physics of pair tunneling prevails. Namely, a thermally excited electron entering the dot at energy $\varepsilon$ causes transport of further electrons at energy $\varepsilon-|U|$ before the dot is emptied again.

Next, we demonstrate how the Seebeck coefficient gets modified in the nonlinear regime due to large $\Delta T$ and $V$. This is also relevant below where we discuss the performance of the quantum dot as a thermoelectric device. The nonlinear thermovoltage $S_{\mathrm{nl}}=\left.V\right|_{I=0} / \Delta T$ quantifies the voltage $V=\mu-\mu_{\mathrm{L}}$ required to suppress a charge current $I$ induced by a large temperature difference $\Delta T=T_{\mathrm{L}}-T$ across the junction.
We have previously shown ${ }^{16}$ the nonlinear current to take the compact form,

$$
I=\frac{\gamma_{\mathrm{L}} \gamma_{\mathrm{R}}}{\gamma_{\mathrm{L}}+\gamma_{\mathrm{R}}}\left(n_{\mathrm{L}}-n_{\mathrm{R}}\right)
$$

in the wideband limit $\left(\Gamma_{\varepsilon \alpha}=\Gamma_{U \alpha}=\Gamma_{\alpha}\right)$. This depends on the difference between equilibrium occupations $n_{\mathrm{R}}=n$ and $n_{\mathrm{L}}=\left.n\right|_{\mu, T \rightarrow \mu_{\mathrm{L}}, T_{\mathrm{L}}}$ and on the energy-level dependent charge relaxation rates ${ }^{83,39} \gamma_{\mathrm{R} / \mathrm{L}}$ $=\Gamma_{\mathrm{R} / \mathrm{L}}\left[1+f_{\mathrm{R} / \mathrm{L}}(\varepsilon)-f_{\mathrm{R} / \mathrm{L}}(\varepsilon-|U|)\right] / 2>0$. Both the occupations and relaxation rates can be understood as if the dot was coupled only to the right or left lead; the symbols $f_{\mathrm{R}}(x)=f(x)$ and $f_{\mathrm{L}}(x)$ $=\left.f(x)\right|_{\mu, T \rightarrow \mu_{\mathrm{L}}, T_{\mathrm{L}}}$ denote the corresponding Fermi functions. Setting $I=0$ while keeping the potential $\mu_{\mathrm{R}}=\mu$ and temperature $T_{\mathrm{R}}=T$ fixed, Eq. (10) yields the helpful analytical result for the thermovoltage, ${ }^{16}$

$$
\begin{aligned}
S_{\mathrm{nl}} \times T= & \varepsilon-|U|-\frac{T+\Delta T}{\Delta T / T} \\
& \times \ln \left[\frac{1-n_{\mathrm{d}}+\sqrt{\left(1-n_{\mathrm{d}}\right)^{2}+\exp \left[-\frac{|U| \Delta T}{T(T+\Delta T)}\right] n_{\mathrm{d}}\left(2-n_{\mathrm{d}}\right)}}{2-n_{\mathrm{d}}}\right],
\end{aligned}
$$

again expressed in terms of the dual occupation number $n_{\mathrm{d}}$.

Figure 3 shows $S_{\mathrm{nl}} \times T$ and its $\varepsilon$-derivative ${ }^{40}$ as a function of level position $\varepsilon$ and temperature difference $\Delta T>0$. As expected, the zero crossing at the particle-hole symmetric point $\varepsilon=|U| / 2$ persists. Importantly, the counter intuitive features at $\varepsilon=0,|U|$ also continue to exist. This is indeed suggested by Eq. (11), in which the $\varepsilon$-dependence enters entirely through the dual occupation $n_{\mathrm{d}}$ determined by repulsive interaction. Specifically, in Fig. 3(c), an increasing $\Delta T$ transforms the steps at $\varepsilon=0,|U|$ between the three regimes of $S_{\mathrm{nl}}(\varepsilon) \times T$ with equal $\varepsilon$-slopes into temperature-broadened transitions between three regimes of different slopes. For $\varepsilon<0$ and $\varepsilon>|U|, S_{\mathrm{nl}}(\varepsilon) \times T$ still grows with a slope of 1 as function of $\varepsilon$, just as the Seebeck coefficient $S(\varepsilon) \times T$ [see Fig. 1(b)]. This again reflects that transport is effectively governed by single-particle physics (see above discussion on energy-dependent couplings). For levels $0<\varepsilon<|U|$ at which twoparticle effects are relevant, a linear $\varepsilon$-dependence of $S_{\mathrm{nl}} \times T$ with a larger slope of $\sim 2$ emerges, as can be qualitatively understood from an analysis of the nonlinear charge current $(10)$ : a small $T \ll|U|$ and a large $\Delta T \gtrsim|U|$ correspond to a sharp two-particle transition of $n_{\mathrm{R}}=2 \rightarrow 0$, yet a smooth behavior of $n_{\mathrm{L}}$ as a function of $\varepsilon$ around

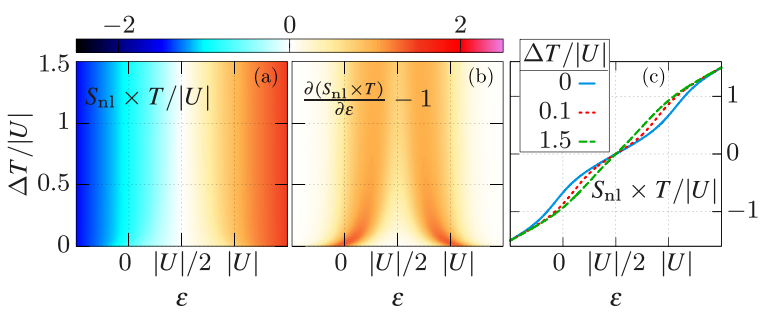

FIG. 3. Nonlinear thermovoltage [(a) and (c)] and its derivative (b) as a function of dot level $\varepsilon$ and temperature gradient $\Delta T /|U|$. We take $T=|U| / 10$ and $\Gamma_{L}, \Gamma_{R}$ that are energy-independent. 
$\varepsilon=|U| / 2$. Consequently, fulfilling $n_{\mathrm{L}}=n_{\mathrm{R}}$ to achieve $I=0$ for fixed $\varepsilon$ and $\mu$ requires a relatively large shift of $V=\mu-\mu_{\mathrm{L}}$. In particular, the slope of 2 of $S_{\text {nl }}(\varepsilon) \times T=\left.\frac{V T}{\Delta T}\right|_{I=0}$ in the limit $\Delta T / T \gg 1$ reflects the sharp change of $n_{\mathrm{R}}$ by 2 at $\varepsilon=|U| / 2$ due to attractive interaction [see Fig. 1(c)].

Finally, let us consider the power output of the dot. As is wellknown, ${ }^{41-44}$ a sharp spectral resonance of a conductor is beneficial for its thermoelectric performance. Hence, quantum dots have been studied as thermoelectric elements operated in the nonlinear regime of large temperature and voltage biases, both theoretically, $8,12,16$ and experimentally. ${ }^{11,20}$ We now show that also in the presence of strong attractive interaction, finite power output is possible at high efficiencies.

We study the power output $P=I \times V$ with current $I$ given by Eq. (10) as well as the efficiency $\eta=P / J$, where $J$ is the heat current out of the left (hot) reservoir. Analytical expressions for $J$ are derived in Ref. 16 for a generic onsite interaction. In Fig. 4(a), we show $P$ and $\eta$ as a function of $V$ for $\Delta T=-|U|, \Gamma_{L}=\Gamma_{R}=\Gamma$ and for an $\varepsilon=1.23|U|$ in the vicinity of the crossover between single- and two-particle regimes, optimized for maximal power output. The power clearly behaves nonmonotonically with a peak at $|V| \approx \Delta T$. Efficiency $\eta$ increases with voltage and assumes about 0.6 times the Carnot efficiency $\eta_{\mathrm{C}}=1-T_{\mathrm{R}} / T_{\mathrm{L}}$ at maximum power. These efficiencies are sizable at finite power output, as can clearly be seen in Fig. 4(b), where $\eta$ and $P$ are shown for several temperature differences $\Delta T$. These also show that efficiencies reach the Carnot limit when power is suppressed at large voltages. ${ }^{45}$

Figures 4(c) and 4(d) show the power $P_{\max }$ maximized over $V$ at otherwise fixed parameters and the efficiency $\eta_{P_{\max }}$ at $P_{\max }$. Extended regions of $\varepsilon$ - and $\Delta T$-values have sizable power $P_{\max } \gtrsim \Gamma T$. Interestingly, Fig. 4(c) shows that the maximum power is fully suppressed only at $\varepsilon=|U| / 2$. This can be understood by the fact that the
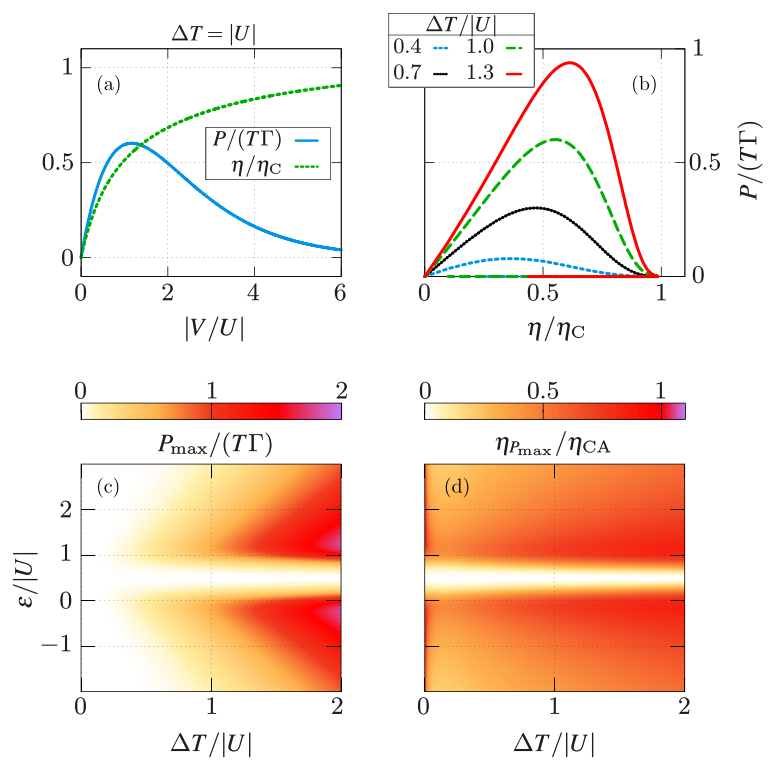

FIG. 4. (a) Power $P$ and efficiency $\eta$ as a function of the voltage bias $V$ at the fixed level position, $\varepsilon=1.23|U|, \Delta T=|U|, T=|U| / 10$. (b) Efficiency vs power at the fixed level position, $\varepsilon=1.23|U|$ for different temperature biases $\Delta T$. (c) Power $P_{\max }$ maximized over $V$. (d) Efficiency $\eta_{P_{\max }}$ at maximum power. In all panels, we set $\Gamma_{L}=\Gamma_{R}=\Gamma$. nonlinear thermovoltage $S_{\text {nl }}$ only disappears at this level position, as shown above. However, in the whole two-particle regime, $0<\varepsilon<|U|$, the power is small compared to the single-particle regime, $\varepsilon<0$ and $\varepsilon>|U|$. The reason is that the charge relaxation rate $\gamma_{R}$ entering the current, Eq. (10), is suppressed in this regime by attractive Coulomb blockade. ${ }^{25}$ This leads to low power production.

Important for the performance of the attractive quantum dot as a heat engine is our finding that the efficiency at maximum output power is on the order of the Curzon-Ahlborn bound, ${ }^{46} \eta_{\mathrm{CA}}=1-\sqrt{T_{\mathrm{R}} / T_{\mathrm{L}}}$, in the whole range in which the output power is sizable. It even reaches this bound close to the dual resonances, $\varepsilon=0,|U|$, namely, the level positions at which $n_{\mathrm{d}}$ obtained from the dual mapping changes by 1 . It is also remarkable that for the finite power output, prominent features appear at the resonances of the dual repulsive model.

To conclude, we have analyzed the thermoelectric response of a weakly coupled, single-level quantum dot with attractive interaction together with its performance as a steady-state heat engine. The presented results are expected to be important for future experiments aiming to characterize systems with strong attractive onsite interaction. At the same time, they demonstrate that nanodevices based on quantum dots with attractive interaction can also efficiently convert heat into work.

The most relevant qualitative features that we found are (i) two stepped features in the Seebeck coefficient $S$ instead of one, unexpectedly located at the positions for resonances of a repulsive dot (Fig. 1). (ii) Nonlinear Seebeck coefficient $S_{\text {nl }}$ is constant up to sizeable thermal bias (Fig. 3). (iii) Sharp dip in the maximum power and efficiency at the position expected for the attractive dot (Fig. 4). We anticipate these effects in state-of-the-art experiments as, e.g., in quantum dots defined at an oxide interface ${ }^{4}$ where clear features of attractive interaction have already been seen in voltage-driven charge transport measurements. Here, applying a temperature bias would allow to verify our predictions, in particular, due to the available electrical control over the level position. In contrast, nanostructures such as in Ref. 3 allow the magnitude and sign of the real interaction to be altered. This would enable a direct comparison between attractive and real repulsive quantum dots. Predictions for such a comparison are outlined in the supplementary material. However, these setups would need to be extended to allow for transport measurements.

The remarkable appearance of prominent features at level positions characteristic of a repulsive quantum dot was rationalized with a dual mapping emerging from a dissipative symmetry for master equations. For attractive quantum dots, the role of this dual mapping is particularly important: The dual features with respect to the original attractive system do not appear at special positions, where, e.g., particle-hole symmetry imposes restrictions. Their prominent role could hence not have been predicted straightforwardly in another way.

See the supplementary material for a comparison to a quantum dot with real repulsive interaction.

We acknowledge financial support from the Knut and Alice Wallenberg foundation and the Swedish VR (J.Sp., J.Sc.) and the Danish National Research Foundation (J.Sc.).

\section{DATA AVAILABILITY}

The data that support the findings of this study are available within this article. 


\section{REFERENCES}

${ }^{1}$ M. R. Butler, B. Movaghar, T. J. Marks, and M. A. Ratner, "Electron pairing in designer materials: A novel strategy for a negative effective Hubbard U," Nano Lett. 15, 1597-1602 (2015).

${ }^{2}$ G. Cheng, M. Tomczyk, S. Lu, J. P. Veazey, M. Huang, P. Irvin, S. Ryu, H. Lee, C.-B. Eom, C. S. Hellberg, and J. Levy, "Electron pairing without superconductivity," Nature 521, 196 (2015).

${ }^{3}$ A. Hamo, A. Benyamini, I. Shapir, I. Khivrich, J. Waissman, K. Kaasbjerg, Y. Oreg, F. von Oppen, and S. Ilani, "Electron attraction mediated by Coulomb repulsion," Nature 535, 395 (2016).

${ }^{4}$ G. E. D. K. Prawiroatmodjo, M. Leijnse, F. Trier, Y. Chen, D. V. Christensen, M. von Soosten, N. Pryds, and T. S. Jespersen, "Transport and excitations in a negativeU quantum dot at the $\mathrm{LaAlO}_{3} / \mathrm{SrTiO}_{3}$ interface," Nat. Commun. 8, 395 (2017).

${ }^{5}$ J. Koch, M. E. Raikh, and F. von Oppen, "Pair tunneling through single molecules,” Phys. Rev. Lett. 96, 056803 (2006).

${ }^{6}$ E. Sela, H.-S. Sim, Y. Oreg, M. E. Raikh, and F. von Oppen, "Electron-pair resonance in the Coulomb blockade," Phys. Rev. Lett. 100, 056809 (2008).

${ }^{7}$ M. Leijnse, M. R. Wegewijs, and M. H. Hettler, "Pair tunneling resonance in the single-electron transport regime," Phys. Rev. Lett. 103, 156803 (2009).

${ }^{8}$ M. Esposito, K. Lindenberg, and C. Van den Broeck, "Thermoelectric efficiency at maximum power in a quantum dot," Europhys. Lett. 85, 60010 (2009).

${ }^{9}$ M. Leijnse, M. R. Wegewijs, and K. Flensberg, "Nonlinear thermoelectric properties of molecular junctions with vibrational coupling," Phys. Rev. B 82, 045412 (2010).

${ }^{10} \mathrm{R}$. Sánchez and M. Büttiker, "Optimal energy quanta to current conversion," Phys. Rev. B 83, 085428 (2011).

${ }^{11}$ S. F. Svensson, E. A. Hoffmann, N. Nakpathomkun, P. M. Wu, H. Q. Xu, H. A. Nilsson, D. Sánchez, V. Kashcheyevs, and H. Linke, "Nonlinear thermovoltage and thermocurrent in quantum dots," New J. Phys. 15, 105011 (2013).

${ }^{12}$ D. M. Kennes, D. Schuricht, and V. Meden, "Efficiency and power of a thermoelectric quantum dot device," Europhys. Lett. 102, 57003 (2013).

${ }^{13}$ B. Sothmann, R. Sánchez, and A. N. Jordan, "Thermoelectric energy harvesting with quantum dots," Nanotechnology 26, 032001 (2015).

${ }^{14}$ A. Svilans, A. M. Burke, S. F. Svensson, M. Leijnse, and H. Linke, "Nonlinear thermoelectric response due to energy-dependent transport properties of a quantum dot," Physica E 82, 34-38 (2016).

${ }^{15} \mathrm{D}$. Sánchez and R. López, "Nonlinear phenomena in quantum thermoelectrics and heat," C. R. Phys. 17, 1060-1071 (2016).

${ }^{16}$ J. Schulenborg, A. D. Marco, J. Vanherck, M. R. Wegewijs, and J. Splettstoesser, "Thermoelectrics of interacting nanosystems-Exploiting superselection instead of time-reversal symmetry," Entropy 19, 668 (2017).

${ }^{17}$ N. Walldorf, A.-P. Jauho, and K. Kaasbjerg, "Thermoelectrics in Coulombcoupled quantum dots: Cotunneling and energy-dependent lead couplings," Phys. Rev. B 96, 115415 (2017).

${ }^{18}$ P. A. Erdman, F. Mazza, R. Bosisio, G. Benenti, R. Fazio, and F. Taddei, "Thermoelectric properties of an interacting quantum dot based heat engine," Phys. Rev. B 95, 245432 (2017).

${ }^{19}$ J. Schulenborg, J. Splettstoesser, and M. R. Wegewijs, "Duality for open fermion systems: Energy-dependent weak coupling and quantum master equations," Phys. Rev. B 98, 235405 (2018)

${ }^{20} \mathrm{M}$. Josefsson, A. Svilans, A. M. Burke, E. A. Hoffmann, S. Fahlvik, C. Thelander, M. Leijnse, and H. Linke, "A quantum-dot heat engine operating close to the thermodynamic efficiency limits,” Nat. Nanotechnol. 13, 920-924 (2018).

${ }^{21}$ F. Hartmann, P. Pfeffer, S. Höfling, M. Kamp, and L. Worschech, "Voltage fluctuation to current converter with Coulomb-coupled quantum dots," Phys. Rev. Lett. 114, 146805 (2015)

${ }^{22}$ B. Roche, P. Roulleau, T. Jullien, Y. Jompol, I. Farrer, D. A. Ritchie, and D. C. Glattli, "Harvesting dissipated energy with a mesoscopic ratchet," Nat. Commun. 6, 6738 (2015).

${ }^{23}$ H. Thierschmann, R. Sánchez, B. Sothmann, F. Arnold, C. Heyn, W. Hansen, H. Buhmann, and L. W. Molenkamp, "Three-terminal energy harvester with coupled quantum dots,” Nat. Nanotechnol. 10, 854 (2015).

${ }^{24}$ H. Thierschmann, R. Sánchez, B. Sothmann, H. Buhmann, and L. W. Molenkamp, "Thermoelectrics with Coulomb-coupled quantum dots," C. R. Phys. 17, 1109-1122 (2016).
${ }^{25}$ J. Schulenborg, R. B. Saptsov, F. Haupt, J. Splettstoesser, and M. R. Wegewijs, "Fermion-parity duality and energy relaxation in interacting open systems," Phys. Rev. B 93, 081411 (2016).

${ }^{26}$ E. Kleinherbers, P. Stegmann, and J. König, "Revealing attractive electron-electron interaction in a quantum dot by full counting statistics," New J. Phys. 20, 073023 (2018).

${ }^{27}$ B. A. Placke, T. Pluecker, J. Splettstoesser, and M. R. Wegewijs, "Attractive and driven interactions in quantum dots: Mechanisms for geometric pumping," Phys. Rev. B 98, 085307 (2018).

${ }^{28}$ V. Bruch, K. Nestmann, J. Schulenborg, and M. R. Wegewijs "Fermionic duality: General symmetry of open systems with strong dissipation and memory" (to be published).

${ }^{29} \mathrm{Up}$ to linear order in the couplings $\Gamma_{\alpha}$, the dual model is a physically valid model and can be interpreted physically. This breaks down in higher-than-linear orders in $\Gamma_{\alpha}$, but the duality remains useful. ${ }^{28}$

${ }^{30}$ C. W. J. Beenakker and A. A. M. Staring, "Theory of the thermopower of a quantum dot," Phys. Rev. B 46, 9667 (1992).

${ }^{31}$ A. A. M. Staring, L. W. Molenkamp, B. W. Alphenaar, H. van Houten, O. J. A. Buyk, M. A. A. Mabesoone, C. W. J. Beenakker, and C. T. Foxon, "Coulomb-blockade oscillations in the thermopower of a quantum dot," Europhys. Lett. 22, 57 (1993).

${ }^{32}$ A. S. Dzurak, C. G. Smith, M. Pepper, D. A. Ritchie, J. E. F. Frost, G. A. C. Jones, and D. G. Hasko, "Observation of Coulomb blockade oscillations in the thermopower of a quantum dot," Solid State Commun. 87, 1145-1149 (1993).

${ }^{33}$ The relation $\delta n_{\mathrm{d}}^{2}=T \partial n_{\mathrm{d}} / \partial \varepsilon$ is proven in Ref. 16.

${ }^{34}$ M. M. Deshmukh, E. Bonet, A. N. Pasupathy, and D. C. Ralph, "Equilibrium and nonequilibrium electron tunneling via discrete quantum states," Phys. Rev. B 65, 073301 (2002).

${ }^{35}$ E. Bonet, M. M. Deshmukh, and D. C. Ralph, "Solving rate equations for electron tunneling via discrete quantum states," Phys. Rev. B 65, 045317 (2002).

${ }^{36}$ S. Juergens, F. Haupt, M. Moskalets, and J. Splettstoesser, "Thermoelectric performance of a driven double quantum dot," Phys. Rev. B 87, 245423 (2013).

${ }^{37} \Lambda$ actually depends on $\varepsilon$ via $\Gamma_{\alpha}(E)$ [Eq. (7)]. In Fig. 2, $\varepsilon$ is swept while adjusting $\Gamma_{\alpha}(E)$ to keep $\Lambda$ constant. However, as Ref. 19 has shown, an $\varepsilon$-sweep that keeps $\Lambda$ constant is achieved without adjusting the couplings for an exponential $\Gamma_{\alpha}(E) \sim \exp \left(\left(E-E_{0}\right) / D\right)$. Such an energy profile has proven to be a reasonable assumption in analyzing several previous experiments on few-electron transport from quantum dots. ${ }^{47-50}$

${ }^{38}$ J. Splettstoesser, M. Governale, J. König, and M. Büttiker, "Charge and spin dynamics in interacting quantum dots,” Phys. Rev. B 81, 165318 (2010).

${ }^{39}$ J. Vanherck, J. Schulenborg, R. B. Saptsov, J. Splettstoesser, and M. R. Wegewijs, "Relaxation of quantum dots in a magnetic field at finite bias: Charge, spin, and heat currents," Phys. Status Solidi B 254, 1600614 (2017).

${ }^{40}$ The derivative can be straightforwardly analytically obtained from Eq. (11).

${ }^{41}$ G. D. Mahan and J. O. Sofo, "The best thermoelectric," Proc. Natl. Acad. Sci. U. S. A. 93, 7436-7439 (1996).

${ }^{42}$ L. D. Hicks and M. S. Dresselhaus, "Effect of quantum-well structures on the thermoelectric figure of merit," Phys. Rev. B 47, 12727-12731 (1993).

${ }^{43}$ L. D. Hicks and M. S. Dresselhaus, "Thermoelectric figure of merit of a onedimensional conductor,” Phys. Rev. B 47, 16631-16634 (1993).

${ }^{44}$ T. E. Humphrey and H. Linke, "Reversible thermoelectric nanomaterials," Phys. Rev. Lett. 94, 096601 (2005).

${ }^{45}$ The sharp feature at low power and high efficiencies stems from the exponential tails of $n_{\mathrm{L} / \mathrm{R}}$, prohibiting an exactly suppressed current. We expect this to be smoothed by here neglected higher-order tunneling effects.

${ }^{46}$ F. L. Curzon and B. Ahlborn, "Efficiency of a Carnot engine at maximum power output," Am. J. Phys. 43, 22 (1975).

${ }^{47}$ K. A. Matveev and L. I. Glazman, "Coulomb blockade of activated conduction," Phys. Rev. B 54, 10339-10341 (1996).

${ }^{48}$ N. M. Zimmerman, E. Hourdakis, Y. Ono, A. Fujiwara, and Y. Takahashi, "Error mechanisms and rates in tunable-barrier single-electron turnstiles and charge-coupled devices," J. Appl. Phys. 96, 5254-5266 (2004).

${ }^{49}$ J. D. Fletcher, M. Kataoka, S. P. Giblin, S. Park, H.-S. Sim, P. See, D. A. Ritchie, J. P. Griffiths, G. A. C. Jones, H. E. Beere, and T. J. B. M. Janssen, "Stabilization of single-electron pumps by high magnetic fields," Phys. Rev. B 86, 155311 (2012).

${ }^{50} \mathrm{~B}$. Kaestner and V. Kashcheyevs, "Non-adiabatic quantized charge pumping with tunable-barrier quantum dots: A review of current progress," Rep. Prog. Phys. 78, 103901 (2015). 